\title{
Una carrera hacia los bordes de la sociedad
}

\author{
Marcelo Berho C. ${ }^{*}$ \\ "corro contra la vida... corro contra dios", \\ Eduardo Quifafi
}

\begin{abstract}
This is a reflection of the anthropological phenomenom homelessness that arises from the analysis of urban marginality and the homeless. Ethnography provides the basis of this reflection. In this sense, I am trying to frame the fieldwork material in a new context using a new matrix and taking into account suitable theoretical notions relative to the case. Important terms, i. e., homeless/homelessness, stigmal stigmatization, to be insideloutside, moral career.
\end{abstract}

\section{Resumen}

Esta es una reflexión en torno a un problema de fondo en el análisis antropológico de una forma de marginalidad urbana, la vagabundancia, y de quienes viven de esa forma, los vagabundos. La fuente básica de este relevo reflexivo es la de la etnografia. En este sentido, el material de campo es colocado en un nuevo contexto, el de la escritura ensayística, en el que puede adquirir nuevos matices dependiendo de las vinculaciones que puedan establecerse entre los diversos materiales empíricos, teniendo en cuenta algunas nociones teóricas y conceptuales adecuadas al caso.

\section{La estigmatización del vagabundo}

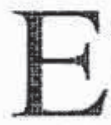
n su libro Estigma el sociólogo de Chicago Erving Goffman advierte, entre otras cosas, el rasgo moral de todo proceso de estigmatización en que las personas son individualizadas y llegan a adquirir una identidad deteriorada de acuerdo a la simbolización de algunos atributos culturalmente percibidos como negativos y definidos de este modo en diversas situaciones sociales. En este proceso, el estigmatizado adquiere, según Goffman (1970), una especie de capacidad protectiva (1993) que, en el drama de la interacción social, se manifiesta de manera progresiva como un abrazo del rol socialmente asignado de quien proyecta uno o varios estigmas.

Este proceso de abrazos de roles estigmatizantes no es otra cosa que la búsqueda personal unida a la impugnación social por alcanzar y expresar la coherencia necesaria entre lo que son las expectativas de rol y el comportamiento efectivamente expresado.

En este dominio de la experiencia simbólica, el comportamiento del vagabundo adquiere una connotación bastante especial debico a que precisamente él (tal vez más que nadie) puede ađquirir esta coherencia identitaria a la vez que negarla llevando una existencia que se autocomprende como fuera del mundo de la vida social.

Como Goffman centró su preocupación en trazar los contornos generales del estigma, no se ocupa de las implicaciones socioculturales del comportamiento y los símbolos estigmatizantes. La etnografía del vagabundo, esbozada hace alrededor de un año atrás (Berho, 1998a), ha permitido comprender este último componente que en principio se vincula a la idea -como hubiese dicho algún interaccionista simbólico de la época- de las cameras morales de personas estigmatizadas. 
Y, es que, una de las dimensiones que, según be ido descubriendo, mcjor permiten hacer inteligibles las vidas de los vagabundos es la dimensión moral de su condición vital. ${ }^{1}$ Moral contextualizada y localizable tanto en lugares como en voces, gestos y acciones. Moral que se opone al resto de las morales porque ninguna deja al individuo ir en su contra. Moral que se transgrede a sí misma en su propia manifestación.

Puede decirse que, en el contexto de la ciudad de Temuco, es importante la presencia de algunas formas de vagabundancia a su vez, que de quienes cncaran la vida desde esas posibilidades (fundamentalmente de las formas de vagabundancia en adultos varones de la ciudad. No se han considerado aún las formas que asume el fenómeno en mujeres, niños y/o jóvenes, aunque se asume que éste varía generacionalmente). De hecho, en sitios como la Feria Pinto y en general el barrio Estación, algunos vagabundos llegan a formar parte de una verdadera economía de los intersticios del mercado basada en ciertos lazos situacionales. Se sabe también que otros pueden ser objeto del humor popular, cuando no de la burla, el desprecio y la caridad. Además, existen evidencias etnográficas propias (Berho, 1998b) y de otros etnógrafos locales (Alarcón, 1998) que vinculan a personas y a carreras personales hacia los bordes de la sociedad.

Aquí en la ciudad: en la Feria, la Estación y sus alrededores, como en otros sitios, una avenida cualquiera, debajo del puente, un sitio eriazo, el descampado... los vagabundos son objeto de un tipo de reconocimiento social basado en una sensibilidad caracterizada por la ambigücdad de los mismos estereotipos generalizantes que se construyen para hablar del sujeto y que, no obstantc, actúan como mecanismos de discernimiento moral dentro de la sociedad, pcrmitiendo hacer la diferencia entre qué conductas son más apropiadas que otras y, sobre esta misma base, operar el juego de la integración frente a la exclusión sociocultural.

Ensayaré, entonces -asumiendo que se puede hacer un ejercicio de este tipo-, algo respecto de las tramas reales que constituyen sentidos profundos en las existencias propiamente tales, bajo el entendido de que estas existencias cxisten en un contexto estigmatizante y excluyentc que traza, en gran medida, los caminos a recorrer, así como la forma en que tal condición es asumida. De modo que, ¿cuáles son las tramas socioculturales que constituyen las carreras morales de los vagabundos en la ciudad? Y, ¿de qué modo puede uno hacerlas inteligibles en su complejidad?, son preguntas sobre las que esbozaré algunas ideas.

\section{Estar adentro/estar afuera}

Estas carreras se empiezan, por lo general, después de situaciones vitales de afto costo y honda repercusión social y emocional (muertes, separaciones, abandonos, pérdidas de bienes, ban- carrotas, ruinas, engaños, locuras) o en un contexto de migración e instalación abortado, en que expectativas y deseos se ven ampliamente frustrados y alterados (como on el caso de migrantes rurales). Diversas son las situaciones que pueden licvar a alguien a quedar en la calle, pero lo cierto es quc cuando a ésta se llega una nueva vida puede comenzar, y una antigua quedar atrás. Llegar a la calle y comenzar a vagar en ella señaia el umbral de la iniciación del yagabundo.

Junto con señalar el comienzo de este proceso, lo que más me interesa es dejar constancia de dónde puede llegar quicn, como en el grito desesperado de Quifafi, concibe la vida como una carrera contra la vida misma. Y, elegí esta expresión porque ella contiene, de manera particular, el sentido profundo de la experiencia de vida del vagabundo, al menos del comúnmente conocido como torrante.

El torrante es un vagabundo particular (Berho, 1998a, 1998b) quc expresa una identidad deteriorada igualmente particular, desde el punto de vista de su conducta habitual. Sus mejores escenificaciones identitarias tienen que ver con su prolongadísimo estado de ebriedad. Tal vez la mejor imagen social para representarlo sea la del viejo alcohólico inveterado que ha perdido tođo, mujer, familia, trabajo, bienes, amistades; que mendiga y que puede presentar diversos grados de desvinculación local y comunitaria, aunque él mismo construya y adapte para sí algunos nichos de acción e interacción.

Arturo llegó hace un año aproximadamente a la ciudad. Aunque se vino después de pelearse con su familia, él dice que vino a probar suerte, a ver si encontraba trabajo y se quedaba a vivir acá un tiempo, después del cual supuestamente volvería a casa. Pero (desde entonces) aún no ha encontrado trabajo y' ya ni siquiera quiere buscar uno. Ahora vive en la calle. La Feria es ahora su hogar: Allí hace su día: come, bebe, duerme, divaga.

Cuando Arturo llegó a la ciudad traía sus pesos, que no eran muchos, con los que viviría mientras encontraba algún trabajo y se acomodaba. Como nada de eso pudo concretarse y como se quedó sin plata y sentía que no podía volver a su casa (pues se había venido después de una pelea familiar), Arturo comenzó de a poco a vagar. Primero a vagar, luego a pedir plata. Comenzó a beber todos los días y a tirarse los piqueros (a dormir donde quedara tendido). En la Feria conoció a otros en situación semejante a la suya. Su apariencia fue cambiando. Dejó de asearse, sus barbas crecieron y no le importó mucho andar con la ropa sucia y con mal olor. Su estado de ánimo y su salud también se fueron alterando. Con 28 años, Arturo sufre actualmente de continuos ataques de epilepsia, que se ven fuertemente acentuados por el uso excesivo de alcohol...

1 En la Historia de la tocura en la época olásica, Foucault (1994) Identifica las consciencias de la experiencia de la locura desøe la Edad Media a la época clásica en Europa, afirmando que, en ese cortexto, tal experiencia se ha vinculado fuenemente al mundo moral. "De lo que se trata (afima Foucaurl), es de toda una relación oscura entre la locura y el mal (que inclufa en un mismo saco todo un bestiario imaginativa moral), relación que ya no pasa, corno en tiempos del Renacimienta por todas las potencias sordas del mundo sino por ese poder individual del hombre que es su voluntarf, Ibid., Toma l, p. 219 . 
Así, lentamente, Arturo se fue dejando arrastrar por los dominios del vicio y la resignada oscuridad de la noche. Dejó que el destino se hiciera cargo de su vida. Fue olvidando a los suyos, su lugar de origen, sus antiguas amistades..

Sólo para introducirse en el mundo de las experiencias estas notas dejan entrever la suerte dialćctica de separación/participación que una persona va atravesando en un camino que conduce a una nueva identidad, y a una identidad forjada desde una condición psicosomática que se va distorsionando, a la vez que una condición socioeconómica de precariedad, donde ambas están estrechamente vinculadas y llegan a cristalizarse en una moral sociocuituralmentc divergente y estigmatizante.

Primero queda atrás el cspacio natural y social de origen. El recuerdo que subsiste de antiguas formas de interacción basadas en la vecindad territorial, el parentesco, la ocupación, el sexo o la amistad es gradualmente disuelto según si la persona adopta las máscaras de la divergencia y que a lo más pueden ser objeto de rememoranza de un pasado en que la vida se vivía de manera muy distinta a la actual.

Lucgo se rompe con los vínculos familiares más directos. "Ya me olvidé de quienes eran (mis padres)". "Ellos dejaron de existir para mî". "No sc si estarán vivos o muertos", son afirmaciones que evidencian de distintos modos la separación tanto física como social y afectiva que asume y experimenta el vagabundo en su infortunada carrera por el mundo.

Al decirle a Rain que yo hablaría con su hermano para que lo fuera a ver, él fue muy enfätico en señalar que no quería saber nada de él, pues ya hacía mucho tiempo que se habia ido de la casa y ni siquiera se acordaba cómo era su hermano y cómo era el trato entre ambos, cuando vivian juntos en la infancia...

También:

Estrada me contó que tenía una hijita viviendo en Australia con una hermana de él. Si bien afirmó seguir queriéndola a la distancia reconoció que, a estas alturas, él no podría ser un buen padre: "de adonde con esta facha, hermanito" - me dijo-, "yo y'a estoy viejo y' feo... no podria, igual que no podría acercarme a mi mujer".

Junto con esta pérdida del rol socialmente asignado a partir del orden primario del parentesco y la familia, hay también un olvido del sí mismo social y espontáneo. Más bien, este úitimo el sí mismo espontáneo- tiende a predominar, pero en una versión dislocada del ideal social y cultural que se traduce en autoabandono y decadencia, otras veces en obstinación y resig- nación autocomplaciente, antes que en cultivo del ego y en profundidad moral. ${ }^{2}$ En efecto, una forma ciega, sorda y muda dc consciencia social comienza a ocupar cl puesto de la habitualidad como alguna vez lo hicieron otros rituales, más relacionados a un rango amplio de expectativas de acción e interacción.

Los descuidos del cuerpo y la salud van por esta misma línea. El cambio inicial es muy espeso y atraviesa y va a atravesar progresivamente la totalidad de la experiencia vital de la persona. Veámoslo en algunos apretados ejemplos: Cuando intenté llevar al scrvicio médico del Hospital a Manucl y a Sergio -dos torrantes-, me encontré con varias razones que justificaban el hecho de que no quisieran ir o que me dejaran esperando para acompañarlos...

La vez que llevaría a Manuel para hacerse un chequeo y para que le desinfectaran y sanaran una pierna que, con el paso del tiempo, tenia podrida, tardé al menos una hora en encontrarlo. Cuando lo encontré, Manuel estaba tendido en el suelo, portaba una bolsa muxy fétida donde llevaba pescado que le habian dado en la Feria y a su alrededor pululaban muchas moscas, algunas de las cuales se le posaban en el cuerpo e incluso en la cara... Le dije que venía para llevarlo al médico, y'él en seguida señaló que no iría pues no se lavaba hacía mucho tiempo...

Por su parte,

Sergio (que tiene aún una hemia del tamaño de una pelota) se la pasaba en esos días bebiendo mucho y olvidaba las horas de consulta al médico, decía cosas como "me quedé dornido", "después me vine a acordar". No se acordaba siquiera que andaba con esa hernia inflada. No sentía ni dolor.

En estos casos se tensan no sólo los aspectos relacionados a la voluntad y la preocupación del sí mismo a través de una cconomía del significado del cuerpo sino también otros relacionados a la actitud frente a los sistemas de cuidado. Pcro, quizá lo más importante sean las demarcaciones sociales que se establecen cuando las conductas habituales se desgastan al punto que el cuerpo se pone putrefacto o está a punto de estallar. Hay aquí un cuerpo indiferente consigo mismo, pues su centro está fuera de él: en el autoabandono de la consciencia, a la deriva en una jornada de excesos, en las cantinas, las picás y las borracheras. Todo esto puede expresarse, en última instancia, en alienación. ${ }^{3}$

Recuerdo como Arturo, habiendo ya acumulado cierta experiencia en la calle, fue vivenciando aspectos delirantes y además muy simbólicos con los que él mismo iba danđo sentido a lo que le iba pasando. Esto igualmentc hay que verlo en el contexto de la gran borrachera de la que -como decía- comenzó a participar Arturo, una vez que sus deseos se fucron viendo frus-

2 Como puede ser el caso de un tipo de divergentes sociales positivamente sancionados, come sor los monjes ascéticos o ciertos artistas.

3 La alienación es también un efecto de lo que Taussig (1995) ha denorninado, slguiendo la idea manxista de *La reificación y la concioncia del prolatariado- de Lukács, ula reificación de la

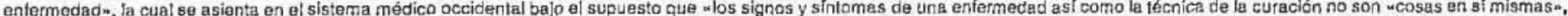
no son sólo biológicas y fisicas sino que son, también, signos de rolaciones sociales disfrazadas como cosas naturates, ocultando sus arigenes en la feciprocidad humana , lbid., $p$. 110. 
trados y comenzó a verse a sí mismo lejos de su antigua manera de vivir...

Una ocasión en que andaba haciendo mis visitas de campo, me encontré con un Arturo desdoblado de si mismo diciendo que el diablo se le habia metido en el cuerpo. El venía de vuelta de un viaje a su casa, en Curacautín, después de estar alejado de los suyos por algo más de seis meses (un viaje que, por lo demás, el propio Arturo no habría hecho si no lo hubiese llevado un voluntario de una las instituciones de apoyo que existen en la ciudad). A esas alturas de su vida Arturo ya hablaba como torrantito y' como el torrantito joven, es decir, un tanto agresivo y sin muchas deferencias, machetero antes que mendigo, bebedor de licor-ron o coñac-antes que de vino...

Esa vez él me contó que como todo eso (lo del viaje) había significado no beber, él se habia vuelto medio loco y que habia tenido una aparición demoniaca en la cual el diablo se introdujo en él enquistándose en su garganta, luego que cruzando por un camino él advirtiera la presencia maligna. Dijo que le costaba trabajo hablar porque justamente el diablo estaba atascado en su garganta... En esos momentos daba la impresión de que Arturo parecía estar fuera de si y actuaba como si una fuerza nueva y rara lo acechara...

Todo esto puede ser no más que un cuadro de abstinencia alcohólica, cargada de delirium tremens y alucinaciones, pero lo cierto es que muestra uno de los punto posibles a los que puede liegar una individualidad desagregada en el contexto de una sociedad que prefigura modiclos de persona según roles y según criterios socioculturales bien distintos que tienen su explicación en la idea del autocontrol, la disciplina, la ética del trabajo y la cultura de la apariencia y la figuración modernas. Quizá por lo misno la existencia de los vagabundos constituya, como en la Edad Media la de los locos y en un primer momento la de los leprosos (Foucault, 1994), parte importante de la consciencia del deber ser y del deber ser así de la propia sociedad de la cual forman parteaunque sea a través de los mecanismos de la exclusión.

Como el de Arturo, hay otros ejemplos. Todos ellos asociados a situaciones fuertes en que la persona experimenta y expresa simbólicamente una caída. La metáfora física de los cuerpos en caída libre capta de alguna manera-física, por cierto- el sentido del proceso. Desde el punto de vista etnográfico, la simbolización más recurrente entre los torrantes de la Feria, y tantos otros, es la que traza equivalencias entre la naturaleza animal del hombre y la situación de vida como vagabundo.

En la mayoría de los casos, el torrante se ve a sí mismo como un perro que vaga en busca de alimento, bebida y un sitio donde descansar y eventualmente dormir. También como los perros, el vagabundo ordena su vida según otra escala temporal y espacial. Sus desplazamientos y la forma en que distribuye sus jornadas cotidianas dependen en gran medida de las exigencias básicas para "mantenerse en pie". Más aún, como señaló Chino hace algún tiempo, "como los perros veo la vida en blanco y negro".

Quien vivencia de este modo su existir no es sino quien ya ha operado los cambios consustanciales de quien abraza el perfil desdibujado de la identidad estigmatizada. Verse como animal y compararse con uno de ellos es el resultado de complejos procesos de autodesconstrucción biográfica en que el desarraigo y la desvinculación, el autoabandono y la anulaciôn de las antiguas formas de socialización no son más que dimensiones duras detrás de las cuales subyacen existcncias indeseables que violentan las formas sociales más públicamente promovidas y culturalmente simbolizadas (la familia, el trabajo, la escuela, la salud, el bienestar material, la estética de consumo).

En térninos sociales, lo anterior se evidencia en la escasa demanda que hacen algunos vagabundos tespecto del cuidado formal que prestan las instituciones y servicios públicos, siempre en el contexto de Temuco. Este silencio acerca de qué cosas podrían darsc y cómo podrían darse sugiere, al menos en quienes callan precisamente, un perfil dramático desde la perspectiva de la intcgración, del cstar adentro. No tener voz ni querer teneria es una actitud común que puede entenderse a la luz de la distancia que el vagabundo va tomando respecto de los canales de acción e interacción positivamente sancionados de la sociedad. $i$ Es que acaso se puede salir efectivamente de la corriente social y ubicarse fuera de la misma? ¿Quién puede hacerio y en qué contextos?

Para un vagabundo profesional ${ }^{4}$ (Berho, 1998a, 1998b), esto es, para un vagabundo de la soledad y el conformismo existencial de vivir bajo el imponderable de la sobrevivencia corporal y mental desplegadas en acciones y palabras guardadas porque no es muy común llevarlas a colación más que cn los términos de un pensamicnto o una visión, pareciera ser común la posibilidad de trastocar el sentido de tal corriente.

Para Clin la basura no es tal. En ella y a través de ella él le da sentido a una variedad de acciones y en general a su vida. Puede alimentarse por su intermedio, siempre y cuando encuentre algún resto de comida. Puede encontrar materiales que usa para diversos propósitos: para reforzar y proteger el sitio donde duerme; para hacer sus tallados - esto es, un reloj, por ejemplo, con una lata de sardinas o conservas, o collares y pulseras, diversos inventos; para sentirse atado a este lugar... Puede pasar horas tendido entre los desechos que la gente tira en las calles o en el tanque de basura de Millahue. A veces puede parecer una lombriz que saborea el gusto de la tierra. Otras una especie de ermitaño que capta en su más mínimo detalle cada latido de las cosas... De hace tiempo que

4 La calegorla ha sido sugerida por la pslquiatra dra, Rossana Echeverria, con quien tuvimos ocasión de intercambiar algunos puntos de vista durante el Seminario sobre Marginalidad social, discapacidad psiquica y redos, organizado por el CES en septiembre de 199B, 
se fue a vivir debajo del puente, donde pasa un buen tiempo levantando rocas para "desarrollar el don de las extremidades". Una tradición según él mismo que le permite mantenerse vivo, incluso cuando no participe del estilo de vida comúnmente aceptado como tal...

Modos de vida como eslos, capturados en pequeñas notas escritas a vuelo de pájaro, scñalan un trastocamiento al nivel de la esfera del significado sociopolítico del comportamicnto, que puede vincularse a los bordes de la vida social en los cuales pucde -extrañamente, para algunos; miserablemente, para la mayoría- vivirse la vida.

En una entrevista sostenida con Clin (Berho, 1998b) en que le preguntaba si él se sentía parte de esta sociedad, él respondía que por supuesto que se sentía parte, al pasar, al andar por ahí, al existir... Esto puede resultar irónico a simple vista, pucs ocurre que Clin no participa habitualmente de situaciones sociales. De hecho, mi relación con él pudo establecerse después de una serie de contactos y entrevistas muy difíciles (y que tienen especial valor para la metodología de campo). La comunicación estuvo sujeta, por entonces, a la incomodidad de no saber de qué hablar y por qué tencr uno y otro que preguntar y responder respectivamentc. Ambos experimentábamos mucha ansiedad, distancia e inquictud. Cundían silencios.

En este punto particularmente es donde entra el tema dcl poder. Y entra y se exterioriza, igual que en los demás campos de la experiencia, por la vía de los símbolos. En Clin mediante la certeza única de que se vive porque se es capaz de hablar, "desarrollar el lenguaje... cl don de las extremidades". El poder está cn la magia de las palabras y en un cuerpo vivo que continuamente constatan la existencia de la persona con sólo darse cuenta que las cosas pueden ser nombradas.

En vagabundos torrantes como Quifafi, Arturo, Estrada, Chino..., el poder está en figuras de autoridad y poder (Pinochet o Allende, la Madre o el Padre, el Presidente de Estados Unidos, Fidel Castro, el General Contreras, la ex Ministro Mónica Madariaga...) que son transformados en íconos y que han influido o no directamente $\mathrm{cn}$ las vidas personales de quien las nombra. En estos términos, la conducta pasada o actual (Quifafi y Estrada fueron perseguidos durante los primeros años de la Dictadura, Arturo presionado por sus padres y Chino protagonista de una historia de mejor final en que la Ministro lo invita a Estados Unidos) puede estar subordinada a distintas significaciones de! poder.

Interesante es ver que estas simbolizaciones representan los lugares que pueden ocuparse en las carreras de la infamia (Foucault, 1993). Los torrantitos y sus historias llenas de personalidades vívidas e intensas (que incluyen a personajes de los más diversos medios, deportistas, artistas, escritores, bandidos, autoridades, etc.) y colmadas de alusiones en que ellos mismos cstán en el centro de los acontecimientos, mucstran los nudos que aún mantienen con la sociedad. Nudos quc, en vagabundos como Clin, son mucho menos evidentes y que, en cualquier caso, operan en otro sentido. En el primer caso, hay una referencia resbaladiza a la sociedad. En el otro, una mueca extemporánea en la cuerda floja de la existencia. Y, es que, en ambos casos, se desarrollan estilos distintos de separación/participación. Las carreras pueden seguir caminos distintos y hacerse bajo otras referencias.

Similarmente, pueden comprenderse las actitudes refractarias hacia el trabajo. El mismo Arturo nos to ha dicho sin palabras cuando, luego de permanecer diez días en una comunidad terapéutica ${ }^{5}$ con el fin de iniciar un proceso rebabilitatorio, decidió tomar sus cosas y salir disparado del establecimiento. La razón, igual que en los casos de varios hombres que han pasado por un proceso similar -de desintoxicación y rehabilitación-, ha tenido que ver con la promoción de valores que reproducen relaciones asimétricas.

Autodisciplina, disposición al trabajo y a las tarcas domésticas -campos propios de los regímenes existentes- son percibidas por el torrante como asuntos que no debieran ser obligatorios ni constrictivos. Quien obliga a hacerlo, aun indirectamentc, no vale la pena. En los casos más acentuados, en que hay pronunciamiento respecto a la acción de mendigar -viejos que lievan años torranteando, reproduciendo una forma de vida-, esta acción es la alternativa frente al hecho de tener un patrón o un jefe, o tener que seguir, al menos, órdenes de otros.

Pero, en estas actitudes hay referencialidad a antiguas instituciones, a diferencia de un vagabundo que, como Clin, queda desde muy pequeño sujeto a una vida de caminante, una especie de gitano ${ }^{6}$ solitario que se inventa la vida él solo, porque carece de las referencias primordiales que la ordenan: sus padres mucren cuando él tienc ocho ó nueve años, a esa misma edad dcja de estudiar y en adelante sus asociaciones son mínimas. En adelante saldrá a caminar por los campos, viviendo de lo que le da la gente o bien comiendo de la frula que va encontrando en sus andares... Como escribió Rimbaud en su poema Vagabundos: "...andábamos errantes, / alimentándonos del vino de las cavernas/y de la galleta del mundo...".

Así, dadas estas manifestaciones simbólicas y dadas las distintas posibilidades con que el vagabundo encara la realidad, los conceptos, las figuras y las formas socioculturales del poder picrden el contenido que suclen tener como conceptos, figuras y formas dadas por garantizadas para transfigurarse, adquirir nuevos sentidos y hasta anularse... Por ello, trabajar puede incluir, por ejemplo, mendigar, hacer favores a cambio de comida o ropa $-\mathrm{y}$ no únicamente dinero-, o recolectar desechos $\mathrm{y}$ acumularlos.

5 Agradezco, en este punto, las informaciones que me proporcionara Ximena Alarcón (1998) respecto de algunas situaciones reveladoras desde el punto de vista etnográfico que ella misma fue recoglendo dentro de un proceso de seguimiento de un vagabundo a quien se le proporcionaron diversos apoyos a nivel del cuidado. 
Esta manera de mirar las cosas y entenderlas compromete la existencia misma del vagabundo junto con la definición đe ésta. Los pololos, los changuitos, ${ }^{7}$ el empleo de poca monta y las relaciones informales de servicios que circunscriben en gran parte el mundo de la vida pública de los vagabundos torrantes también están los enjambres del vicio-expresan silenciosamente una idea de libertad en que el contacto y la interacción social no se interpretan como aspectos completamente indispensables para vivir. Esta idea se acentúa, aún más, en la medida en que tales manifestaciones se ven reducidas o evitadas. Y ello va en directa relación, a mi juicio, con los atributos marcadamente estigmatizantes que son proyectados por los distintos vagabundos, sear torrantes, alucinatorios o profesionales, a la luz de un rango invisible de significados y funciones morales del estigma.

En general, esto último sc observa significativamente en la presentación de la persona, en las escenificaciones. Una persona con un rostro demacrado, con aliento a alcohol, con costras de suciedad y un intenso hedor alrededor suyo es socialmente percibida de un modo más negativo que una con rostro límpido y sin malos olores, aunque beba, mendigue y duerma en la calle. Para ambos el trato interaccional será diferencial, como pude observarlo en el contexto de la consulta médica:

Después que se atendió Vega (mejor presentado, a pesar de vivir en la calle), vino don Manuel. En la primera consulta el médico se habia mostrado medianamente molesto por el ligero mal olor expelido al instante que Vega se quitara la ropa, pero con la entrada de don Manuel -que tenia una herida agusanada, según la expresión de los propios viejos que lo conocian-pude constatar el hecho de que mientras mejor se presenten las personas (bien vestidas, limpias, sin olor, con deferencia), mejor será la atención que pueda recibir e incluso mejor será la disposición del médico para hacer bien su trabajo. Don Manuel andaba realmente fétido para nuestro olfato, más encima andaba entonado y con tufo a vino pipeño... La primera reacción del médico fue "y por qué lo trae así?... debieron bañarlo primero", gesticulando de modo tal que parecía no caberle en la cabeza el que Manuel anduviera así, y más encima acompañado por mí... De hecho, la atención a don Manuel duró mucho menos que la de Vega, aun cuando era el que tenía mayor urgencia de ser atendido y' tratado. ${ }^{8}$

Por la misma razón que el médico en esa oportunidad reaccionó como lo hizo, Manuel, así como otros, prefieren no tener muchos contactos comunicativos con el mundo institucionalizado. Es como si así, y sólo así, estas personas sostuvieran algo que no es sino un producto de la intersubjetividad que tanto evaden: la categorización y el estereotipo. De este modo queda abierto todo un campo para la libertad individual en que la propia cate- goría de vagabundo y con ella todos los estereotipos que la cortejan están implicados. Ya sea por opción, por autoabandono, desenfreno, indiferencia, locura o mal social, el vagabundo sigue una carrera de descrédito social y descibujación personal.

A través de ella el vagabundo pucde alcanzar una doble posición en el mundo de la vida social: 1) ser un desvinculado, un desarraigado de Ios lazos primarios y secundarios, y 2) ser un emancipado que descubre los grados más bajos e inconmovibles de la existencia humana en el presente.

Ambos elementos se vinculan a la orientación individualista que mueve al vagabundo concreto fuera del comportamiento convencional y de las costumbres. Primero, porque hay que alimentarse, abrigarse y cobijarse. Segundo, porque el corte con los vínculos sociales más vernáculos es percibido como prácticamente irreversible. Y, tercero, porque en esas condiciones no queda más que seguir como sea, "contra la vida o contra dios".

En esta orientación, de nuevo, encontramos matizaciones que permiten establecer diferencias en los modos de vivir como vagabundo a la vez que dimensionar los puntos de fuga en relación a la sociedad. El torrante, por ejemplo, es más gregario que el alucinatorio o que el profesional, en la medida en que estos últimos -sobre todo los alucinatorios- tienen menos capacidad para reciprocar con otras personas. Esto lo ubica -ai torrante- dentro de la carrera moral del descrédito en una posición menos dramática si se compara con quien evita el contacto comunicativo cara a cara.

Figurativamente hablando, se podría decir que Arturo -y otros torrantes- se halla en una situación Jiminal en que circunstancialmente puede ir de un lugar a otro del umbral (si no, basta con recordar tan solo que son y han sido diversas las situaciones en que Arturo y otros torrantes han accedido temporalmente a los servicios de cuidados, sobre todo formales $)^{9} \mathrm{cn}$ tanto que Clin ha traspasado ese umbral del cual al parecer no regresará jamás (debiera evocar, para este caso, una imagen en que para entender qué respondía Clin a una de mis tantas preguntas -en condición de inquisidor- yo me iba acercando a él, mientras él se iba distanciando de mí, manteniendo siempre una brecha...). En estas circunstancias, la definición de la persona desce el punto de vista de la interacción y la referencialidad social parece proporcionar un marco adecuado para afirmar que estas carreras pueden conducir a los límites de la sociedad a través de procesos marcados por distintos modos de separación/participación que quizá en el caso de los torrantes estén más claros que en el de los alucinatorios. Pero, ¿qué categoría puede ser ésta?

A fuerza de asir la variedad fenomenológica de la vagabundancia, esta categoría intenta dar cuenta de aquellos vagabundos que más evidentemente no logran establecer relaciones sociales ni mantener una reciprocidad comunicativa amplia. Esta categoría, junto

7 Términos vemáculos usados para referirse a empleos situacionales de poca monta, aunque están vinculacos a trabajo pesado.

8 Más aủn, đon Manual no siguió siendo tratado mientras pudo haberse hecho algo; falleció en el inviemo de 1999 a causa do una tremenda infección.

9 Este es un ảmbita de mucho interés que aủn no ha podido ser desamollado desde el punto de vista etnográfico, sobre todo tratándose de personas que inician y terminan procesos tehabiltatotios. 
con la del vagabundo profesional, amplían enormemente los horizontes del estar adentro/estar afuera que, como aquí se ha supuesto, trazan estructuralmente los contornos de la vida del vagabundo en la conriente de la vida social. A la luz de esta última categoría etnográfica (analítica) se complica más el hecho de si realmente el vagabundo logra salir de tal conriente de la vida social. El problema es determinar en qué consiste estar afuera de esa corrientc. Para esto quisiera desarrollar únicamente la idea de que, si bien existe toda una desvinculación hacia las referencias sociales primarias (y secundarias), no puede ocurrir lo mismo respecto de la esfera de la cultura. Y, es que, hasta donde he podido indagar, la cultura es el dispositivo básico del que depende incluso una orientación de vida como la que adoptan distintos vagabundos en la ciudad. La pregunta clave ahora es: ssignifica estar afuera de la vida social, estar también afuera de la cultura?, o, más bien: ¿puede vivirse una vida desvinculada de la sociedad sin sostenerse a partir de algunos trazos de cultura?

\section{En los bordes de la sociedad, cultura}

De alguna manera se ha visto que, en las carreras morales que desarrollan los vagabundos, de lo que se trata es de destituir de sus cualidades positivas las identidades prefiguradas que establece la sociedad y que se basan en modelos de rol respectivamente sancionados como ideales e indeseables. Hasta aquí he apuntado, precisamente, algunas de las tramas sociales que tienen lugar en aquellos procesos en que la persona adquiere una identidad estigmatizada. Principalmente, he sugerido que estos procesos tienen que ver con dos situaciones: 1) ruptura y separación con las referencias sociales básicas (en el caso de torrantes como Quifafi, Arturo, Chino, Estrada, Raín, etc.), y 2) una socialización temprana y abruptamente abortada (como en el caso de profesionales como Clin).

Sin embargo, al incorporar la perspectiva de la cultura, entendida esta como un sistema de significados relativamente compartidos acerca de las distintas cosas y dimensiones del mundo que permiten la comunicación humana en diversos niveles, encontramos que a un nivel fundamental es prácticamente imposible llevar una carrera que conduzca afuera de la corriente de la vida social, aun cuando desde la perspectiva estructural sólo veamos separación, disgregación y, aún más, individualismo.

En el examen de la relación entre las carreras morales de los vagabundos y la cultura -en el sentido general con que he presentado ei concepto-, el elemento comunicativo es clave para establecer interpretaciones antropológicas pertinentes. Sin ir más lejos, una carrera moral en que lo que se proyecta es una expresión directa de una identidad estigmatizada no puede escindirse de este elemento comunicativo. Es más, siguiendo a Goffman, «la expresión cumple el papel de transmitir las impresiones del sí mismo» (1993: 265), lo cual quiere decir que en toda existencia que se exprese como tal las personas no pueden prescindir de la exteriorización de un flujo de mensajes con algún tipo de significado.
Cualesquiera que sean estos significados, tanto desde la perspectiva del actor como de su audiencia, lo cierto es que siempre se requiere de algún tipo de dispositivo que los haga manifiestos en la realidad. Como he podido captar en la experiencia de vagabundos alucinatorios (quienes no se ajustan completamente ni a las situaciones 1) ni 2) de las establecidas más arriba), donde la definición de la situación pasa tanto por la pérdida de los vínculos como por una socialización rota, lo único imborrable de esa experiencia es el lenguaje, ya sea hablado, de señales, de insinuaciones, gestos expresivos u otras simbolizaciones más o menos aceptadas... Así, por ejemplo, como ocurre en conversaciones con algunos vagabundos visiblemente insensatos.

En una entrevista que sostuvo Pelayo con una psiquiatra y en la que pude hacer un registro de la misma, pueden verse los giros lingüísticos, las referencias simbólicas y la alegoría con que la productividad delirante de la persona satura las preguntas del especialista estableciendo los límites de la reciprocidad comunicativa $-\mathrm{y}$ apoyando la idea de que en los límites de la sociedad hay cultura:

Dra.: ... ¿qué hace por acá en Temuco?

P: El caballero de allá, Tapia Olivares, me mandó para acá...

Dra.: Ya, ¿por qué?

$P:$ Por represalia, porque resulta que parece que este caballero se le olvidó allá, porque yo había perdido... se me habia hecho tira el papel de la jubilación... entonces yo fui para allá y me dio alojamiento y todo... faltaba que me diera mujer no más... pero entonces yo sabia por la mente que el joven mayor parece que iba por ahi y los otros lolos iban despues y hacian el amor... entonces fui para allá y estábamos en el comedor conversando los dos solos... y entonces yo le dije... cómo tenía que decir porque no se si sabía... le conversé que... por ejemplo si yo quería que él me haga el amor a mí yo no tengo que decirle a él... eso le dije a ése niño... entonces se entusiasmó y me dijo «bueno»... pero resulta que eso es feo, ese caballero, padre o madre lleva para allá a uno como allegado y no es mujer, puh, no es mujer..

O:

Dra.: ¿ Ud. escucha voces?

P: No, ahora no...

Dra: $Y$, antes escuchaba?

$P$ : Sí, algo de voces, sí... -y comienza pícaramente a sonreír.

Dra.: Y, por qué se ríe? Le da risa?

$P:$ No, no me río de usted.. es que resulta que allá en el Hospital de Valparaíso me preguntaban eso de las voces también -continúa riendo.

Dra.: ... No ha escuchado pero antes escuchó, cuando más cabro...

P: No, sí, no, sí.. -se da un tiempo y continúa:- no si resulta que en Quillota todos dicen que el General 
Pinochet me jubiló por loco, pero yo sabía todo, que ganaba cuarentitrés y no si porque me dieron a mí un este... un decreto, yo sabia que se llamaba, por la frase que hago...

Detrás de estas conversaciones hay historias que pueden ser diferentes, pero en las cuales resucna aún cl eco de otros acontecimientos: acontecimientos de importancia para quien los cuenta. De hecho, en la mayor parte de los contextos en que pucden establecerse conversaciones con vagabundos como Pelayo, bullen con intensidad fragmentos de historias personales, nombres de personas, lugares, épocas... Estos referentes simbólicos son, no obstante, reconceptualizados bajo ideas alucinantes y subjetivantes que hacen sucumbir las explicaciones aceptadas por la mayoría como explicaciones «cuerdas». Esto pareciera tener que ver más con una canalización distinta de contenidos culturales localizados antes que con la inexistencia de códigos para la comunicación, como alguien pudo haber supuesto.

Nuevamente, la mejor manera que tengo para darme a entender sobre estos asuntos es a través de la ilustración. Se trata de dos cventos descritos y relatados, cada uno, por dos vagabundos alucinatorios, acerca de cómo llegaron a ser, cada uno, vagabundos -aunque uno de ellos (Pelayo) esté en estos momentos en una nueva situación. ${ }^{10}$

Pelayo dijo que él supo, por la mente, que su casa estaba embrijada y que iba a demorar dos años hasta que se terminara el embrijo... Desde entonces él se fue de Quillota, que era donde vivía, y comenzó a vagar.. Por el camino dijo que había entendido lo que le ocurría al darse cuenta que su cuerpo no estaba entero y que tenía cada una de las partes cambiadas... Por ejemplo, decia, tengo el pulmón de uno, el hígado de otro y así... Dijo también que cuando estuvo enfermo -todavía lo está, de acuerdo a los psiquiatras- él habia buscado por todos lados y que quizá alguien habia llamado a los espíritus para hacerle mal a él...

Y, en el otro caso:

Resulta que él venía de Valdivia donde había hecho el servicio military no sabe cómo se quedó dormido y cuando despertó estaba rodeado de niños en algo parecido a la estación de trenes... Dijo que estos niños lo cuidaron todo el tiempo mientras estuvo en ese lugar.. Después que los niños lo dejaron solo, él no halló qué hacer, se puso muy mal y no sabe que siguió...
Claro que estas son maneras particulares de ver las cosas, nombrarlas y entenderlas y, en esa medida, igualmente que como pueden hacer referencias simbólicas a distintos ámbitos de la realidad, no están exentas de códigos comuricativos. «Todas ellas son experimentadas como algo, lo otro en medio del ser. Emergen efectivamente dentro de un mundo de complejos simbólicos, dentro de dominios de experiencia simbolizada» (Berho, 1998b: 55).

Aquí no se puede salir del lenguaje, aunque sí tras vasijarlo de sus significados habituales. Y, en este nivel, lo que interesa dejar claramente establecido es que, de acuerdo a la evidencia etnográfica disponible, existe la posibilidad de excluirse de las tramas sociales más institucionalizadas (incluyendo los sistcmas de cuidado) $y$, no obstante, permanecer cnredado en los referentes del lenguaje y en las simbolizaciones más genuinas de la cultura.

«Ya se trate -adclantaba ya hace un año- de un mal (como en Pelayo), de un cambio de fisonomía (en Clin), o de lo que sea, éstas son explicaciones comunicables dentro del mundo de la vida social. Y el mundo de la vida social no es otro que el mundo de significados compartidos -por más excéntricas que puedan ser las formas que estos toman en la vida individual de quienes los aplican y los proyectan» (Berho, 1998b: 55).

Si todo esto se quisiera ver desde una visión panorámica, habría que colocar en la base del descrédito al vagabundo alucinatorio, seguido del profesional e inmediatamente del torrante. Esta jerarquía tendría que ver, en última instancia, con los grados de separación/participación del vagabundo en los mundos de la vida social y cultural o, en otras palabras, en el ámbito estructural y en el simbólico de la experiencia. Los contenidos de esos grados estarían dados, a su vez, por diversas marcas estigmatizantes: la desvinculación y el autoabandono, la (hiper)subjetivación de los símbolos y la falta de reciprocidad dc los actos comunicantes. Cada una de estas marcas estigmatizantes se atravicsan y yuxtaponen, a su vez, unas con otras, de modo que las categorías no son fijaciones estáticas sino que responden al contexto general en el que pueden tomar forma las vidas de los vagabundos.

De modo que, recapitulando, estas carreras morales se constituyen, según se ha visto, de diversos modos. En todos ellos está presente, de cualquier forma, la individualidad frente a la sociabilidad. De hecho, diría que este es un rasgo sustantivo on y de todos estos procesos. Puede manifestarse tanto a través de una conducta excesivamente alcohólica -como en cl torrante-, como a través de una obsesivamente alienante -el alucinatorio- o genérica -como en el profesional-. El deterioro social, la fragmentación psíquica y la errancia conductual y verbal conducen a una subjetividad que se escapa de la convención y la moral pública porque encuentra en la deriva de los marcos previamente fijados la zona en que cada cual puede romper los cercos de la circunstancialidad normativa y existir sin la necesidad imperiosa de los discursos y las referencias totalizantes -a cualquier escala. 
El final de esta carrera parece conducir al aniquilamiento de los sentidos más colectivos. Puede verse en el tono desvergonzado $y$ la distancia moral con que un torrante mendiga o insulta a otro; en la inconexión de los referentes o en el trance de palabras sin fondo con que vertiginosamente se expresa un alucinatorio; o en la discontinuidad vivencial sobrccargada de adornos y signos estéticamente descchables de un vagabundo profesional. A su vez, cl final representa el lugar y el tiempo cn que estas identidades pueden, en su deriva y desde su anonimato, desplegarse como taies, sin dificultades.

El vagabundo puede ser, de este modo, entendido como un no-refiejo de lo que todos socioculturalmente deberían ser y hacer. En esta medida, y sólo en esta medida, su final llega hasta los bordes de la vida sociocultural. Desde ahí, él nos interroga y su interrogación apunta a las bases mismas de la existencia humana.

El vagabundo guarda para nosotros una crítica silenciosa de nucstro sistema moral de valores. Al presentarse como un vicioso y autodegradado, un loco delirante o alguien inexorablcmente ocioso y excluido, el vagabundo nos muestra (escandalosamente, para algunos) justamente aquel reverso que no siempre queremos descubrir, lo que ha sido definido social y culturalmente como un no ser, lo ajeno, lo otro en el ser.

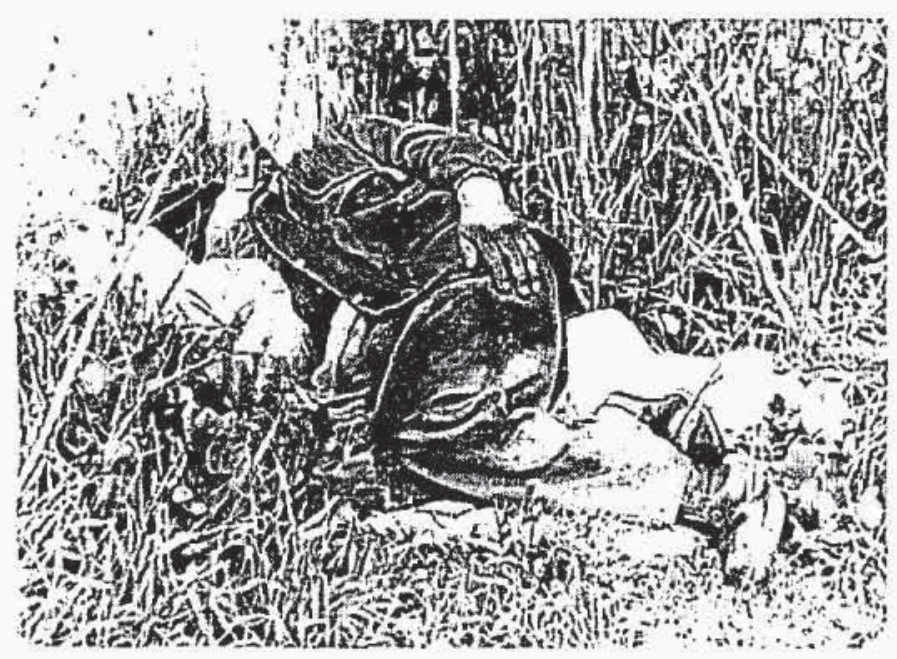

En la imagen, un vagabundo torrante.

\section{La identidad última}

Pero, $i$ cuales son esas bases que, al ser removidas por la sola existencia de los vagabundos, son capaces de turbar sensibilidades y entendimientos dados por garantizados? Ya en los siglos XIV y XV, cuando el vagabundo se confundía con los locos, los ladrones, los mentirosos y los de espíritu débil (Foucault, 1994), existía la creencia de inspiración cristiana bastante extendida por entonces- cn torno a la figura del Hijo Pródigo (Bosing, 1994). Este a su vez era representado a veces por el personaje de El Peregrino quien, imitando a Cristo, renunciaba a este mundo para seguir el ejcmplo del Hijo de
Dios. Lo que garantizaba su existencia cra, sin duda, que al imitar a Jesucristo podía tener la esperanza de atravesar, en su divagar, la noche oscura de este mundo para acceder a las giorias eternas del Paraíso.

Así como en el Renacimiento la imaginación colectiva conectaba al hombre con las potencias de lo Absoluto y, por este medio, le infundía sentido a su propia naturaleza, así también hoy día cxistc un tipo de conexión entre las figuras y representaciones en torno al vagabundo y la coherencia de la experiencia de éste respecto de las mismas.

Así lo advierte el infortunio de las experiencias de tanto torrante, ejemplar e iconográficamente relatado:

Chino dijo que así como él venía de la tierra, terminaria alli mismo. El único que puede verlo a uno y saber que se tiene en mente es el hermano mayor-como le llama a Dios... Dijo que los demás, nadie aqui, puede meterse en la mente de ninguna otra persona, sólo Dios, que está arriba en los cielos... Entonces, muy emocionado, Chino se comparó con Jesucristo diciendo que a él ya le habian puesto una corona de espinas. Mostró entonces su cabeza y pude ver que le faltaba pelo, que más bien ahi no le podia salir pelo... -esto fue un sablazo que me dieron en defensa de un hermano, afirmó. En seguida dijo que ya le habían clavado las manos. Indicó que en la mano derecha habia recibido un balazo y que la izquierda se la había quebrado sin quedar bien. Falta no más que me claven los pies y me crucifiguen... -continuó, resignado.

Pero, ¿pueden ser tan graves esos infortunios como para descuidarse de uno mismo y acceder simbólicamente a una especie de redención? (Me parece que un campo de indudable interés interpretativo al respecto es el de los sistemas de cuidado, entendidos como todas aquellas instituciones, organizaciones y/o grupos cuya razón de ser es el brindar diversas atenciones, apoyos y asistencias dirigidas a grupos más o menos vulnerables de la sociedad.)

Por otro lado, está también la simplicidad profunda de las respuestas de Clin:

«... Cuando nací, digamos, y' todo eso me aconteció... y un día empecé a ejercitar mis extremidades, tenía catorce años, levantaba pesas hasta ahora, porque el cuerpo necesita actividad...

E: El cuerpo necesita mucha actividad?

Clin: Claro, en las extremidades está el lenguaje, para no decaer, digamos...

$E: Y$, el lenguaje, Clin, ¿qué es lo que es?

Clin: Bueno, el lenguaje como desarrollo mental... como también puede ejercitar la persona... en eso me paso todos los días, ejercitándome...

E: ¿...y para eso tú no necesitas vivir en ninguna casa, no cierto? 
Clin: No, ya es costumbre así, como quien dice eso es prehistórico, que han vivido antes de la era moderna, de todo eso me doj' cuenta... no sé, puh, a veces digo "yo soy inventor»... pero a quién aprendí.. asi que sólo digo "respeto al Señor» y todas las noches hago oraciones al Señor y me acuesto y me levanto y quiero estar conforme y me siento conforme...»

En ambas voces lo que está detrás es el mundo de la vida visto por cada quien. La identidad de ambos se basa igualmente $\mathrm{cn}$ haber vivido hasta aquí vidas coherentes a cada acto, a cada expresión, a cada lazo con la vida sociocultural, aunque no sea más que en el dominio de la manifestación dialéctica del reverso de ésta. Ambos están nadanđo en cl mismo mar que nosotros lo hacemos, pero -cada uno a su manera-, la diferencia es que unos nadan a favor y otros en contra de sus olas.

En ambos casos, a su vez, la figura de lo Absoluto aparece para recordarnos la necesidad humanamente cultivada de tal dimensión. Es como si, después de haber llegado a los bordes sociales de la vida misma, no quedara más que Dios y su infinita gracia. Después de haber perdido el rostro social, «sólo Dios sabe»...

En el fondo, la subjetividad, que parece hasta ella misma autoaniquilarse y vaciarse, debe encontrar su sentido último en una expericncia dentro de este mundo. La existencia y la historia de cada existencia son el material con el cual cada identidad logra tomar forma y constituirse, lejos de la convención prefijada y el estilo de vida más generalizado.

Como estas existencias tienden a borrarse desde la perspectiva moral, no queda más que quedar en manos de lo fundamental. Expresiones como: «el Señor Todopoderoso determinará qué pasa con mi vida, si me muern, si sigo viviendo...», o la misma expresión con la cual quise partir esta reflexión: "corro contra la vida... corro contra Dios», permiten captar el sentido de estas afirmaciones.

En un mundo donde necesariamente se requieren habilidades para sobrevivir y "mantenerse en pie», donde las comunicaciones y la interacción pueden continuamente quedar abiertas a su imposibilidad, lo tunico que queda es la propia existencia. Tal vě así, como El Peregrino del Renacimiento, el vagabundo de hoy esté atravesando la oscura noche de su existencia para comprendernos mejor a todos nosotros.

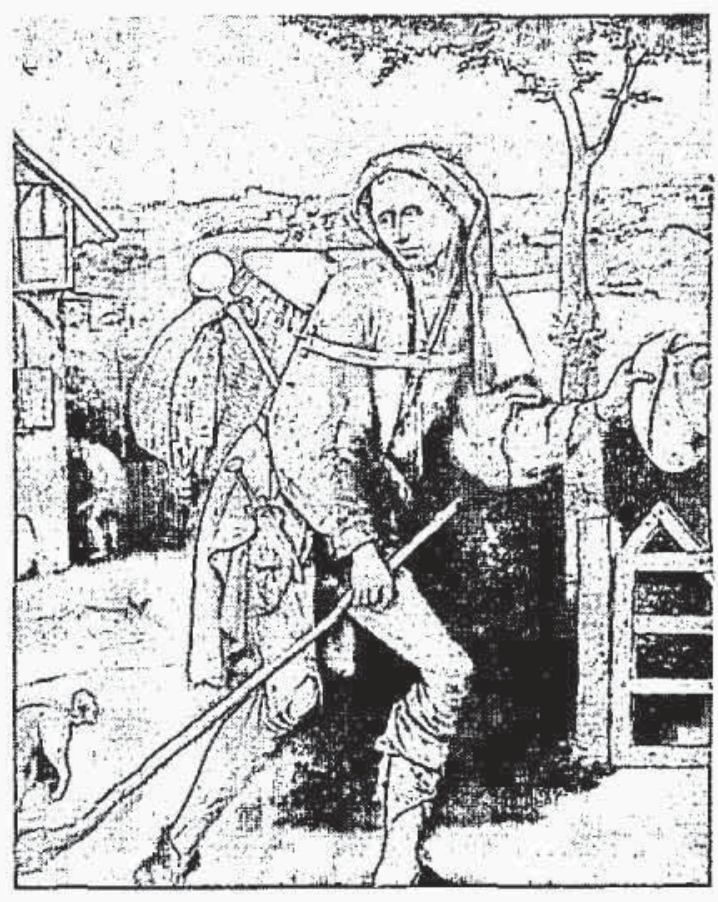

La figura del vagabundo se remonta en la cultura occidental al menos a la Edad Media. Desde entonecs, su definición ba estado dada por diferentes cansciencias. En el Renacimiento se le vinculaba a El Peregrino o E Hijo Pródigo, como muestra la pintura de E. Bosco (1.450 2?-1.516), bajo la creencia que este personaje, nl igual que Cristo, tomaba la plenitud de la salvación espirimal.

\section{Después de la etnografía}

Antes de cerrar este ensayo quisiera dejar enunciadas algunas proposiciones de carácter teórico y metodológico. Ello porque básicamente considero que en antropología uno está necesaria y continuamente yendo $y$ volviendo del contexto a las ideas aun cuando ambos momentos puedan ocurrir simultáneamente $y$ en un sentido como en otro.

Me parece importante dejar claro que el tema o los temas de interés actual no se agotan aquí. La cultura que al parecer subyace a estas carreras sugiere que ésta ha sido, en principio, parte de la cultura global, aunque vaciada de sus contenidos originales de sentido común. Sugierc, por lo tanto, quc es posible también una relación inevitable de la cultura con la subjetividad y, en esta medida, le advierte a la antropología -a sus teorías y sus métodos- respecto de la importancia de incorporar la dimensión individual de la experiencia sociocultural. Ya han habido algunos intentos serios al respecto (Levy, 1992; Le Vine, 1992; Rosaldo, 1992; Geertz, 1973) y llama la atención la poca difusión y điscusión de los mismos en nuestro contexto.

Por la misma razón, estas líneas pucden proporcionar algunos antecedentes relevantes dentro dcl contexto exiguo de construcción teórica en que estamos inmersos, sobre todo si constatamos la presencia incipiente de una antropología más experimental e igualmente revisionista de tradiciones precedentes. 
Esta antropología tiene sus más valiosos trazos en las aproximaciones etnográficas que se están llevando a cabo en el Centro de Estudios Socioculturales, desde su línea de Marginalidad Urbana. A un nivel metodológico, los acercamientos de campo y los seguimientos personales han permitido ir contrastando las categorías de interpretación ya emergentes, tales como los tipos de personas que pueden ser los vagabundos si estos son definidos como torrantes, alucinatorios o profesionales; al igual que iniciar un proceso de contacto y diálogo con instituciones y organizaciones urbanas de apoyo que -se espera-, tienda al cambio sociocultural. Al mismo tiempo, nuevas observaciones y continuas profundizaciones remiten a dar cuenta del potencial analítico e interpretativo de las categorías.

En este sentido, uno de los principales desarrollos a nivel teórico-metodológico ha siđo disponer de categorías capaces de captar en su manifestación el significado posible de lo que se observa, se oye, siente e interpreta. Puede decirse que es el resultado situacional del trabajo de campo realizado desde el año 1998 en varios contextos de la ciudad y, en este sentido, es un esfuerzo dialéctico por delinear los contenidos con los que me parece posible visualizar y aprehender las particularidades existenciales de unos de los más excluidos de la ciudad.

Una manera de cristalizar la identidad deteriorada del vagabundo en Temuco la permitió la constatación de un conjunto polimorfo de voces. Junto a esta polifonía, una estética y una economía de la comunicación igualmente diferenciada. Esta es la consciencia de la presentación del sí mismo, de una escenificación dramática (Goffman, 1993b), que no es únicamente un asunto individual. (No todo el mundo es lo demasiado ciego en nuestro contexto como para no discernir entre una persona que es aprehendida y definida como un alcohólico y otra que es definida como un loco, aun cuando abunden las confusiones.) Se trata, por cierto, de una cuestión que se đefine en el concierto del consenso y la discrepancia intersubjetiva. Tanto como una cuestión de visibilidades y caras (Goffman, 1993b, 1993a), se trata también de un juego de definiciones y construcciones de ideas que pueden o no dar cuenta justa de los significados que intencionalmente o no le otorga la persona a su vida.

De modo que, si bien este sí mismo es autónomo en su individualidad, esto no significa que no haya -como mostraré luegouna interdependencia con la dimensión sociocultural. Emerge así una segunda consciencia del vagabundo. Inteligible a partir de la interacción de éste con el mundo de la vida social, sus formas y sus símbolos. Las rutinas de acción e interacción, la vida cotidiana, los lugares, las palabras, las transacciones y las evitaciones (sociales) son recogidas con el fin de redibujar una imagen densa del sujeto. También, permite dar cuenta de esa variabilidad que aparece ya en la dimensión del sí mismo -el que corre contra la vida, el que se deja llevar por sus cauces y el que la vive complaciente, casi místicamente-, al constatar que, en efecto, el mundo de la vida social que se construye y se dinamiza, varía de un sujeto a otro -el torrante está constantemente jugando con la pareja separación/participación, el alucinatorio apenas logra reciprocar para mantenerse en pie, el profesional, como tal, que nos mira desde el borde de las formas socioculturales, dejándonos atónitos.

En segundo lugar, y a un nivel más teórico, me gustaría señałar la idea de que el comportamiento individual, ni siquiera en los ámbitos más marginales y solitarios de la experiencia humana, es apreciable, no desde una "esencia interna» o "privada», puramente subjetiva y presocial -ajena al mundo social-, sino desde la experiencia en un mundo de significados en que todas las personas están involucradas de manera inevitable. Los significados, según lo ha mostrado Geertz (1973, 1991), son asuntos públicos, se encuentran en una plaza, en una feria o un cine. Y la vida personal, con sus historias, sus cuerpos y sus diferentes maneras de ser, toma forma y se constiturye en términos socioculturales.

Asimismo, los propios individuos aprehenden y transforman interpretativamente los símbolos recibidos. Y, al poder interpretar los símbolos, el individuo puede transformar los significados culturales de acuerdo a su historia y su manera de ser. Como ha sugerido Rosaldo: «Cultural models thus derive from, as they describe, the world in which we live, and at the same time provide a basis for the organization of activities, responses, perceptions, and experiences by the conscious self"' (1992:140).

De modo que, por más en los bordes y a la deriva que se encuentren los individuos respecto del marco normativo del mundo de la vida social -los vagabundos encarnan de maneras variables tal posibilidad-, éstos no podrán estar totalmente afuera, pues los propios modelos (anti)socioculturales que por sus existencias se expresan, son derivaciones oblicuas del mundo en que vivimos. De manera que, «...from the «interpretativist» point of view, does it make sense to claim that individual... are cultural systems cast in miniature» (Ibid,p., 141). A través precisamente de la existencia individual y de la subjetividad del comportamiento es como los símbolos socioculturales llegan a adquirir la forma que adquieren. Así adquieren sentido las historias y maneras individuales en las que, hasta donde he podido ilustrar, se manifiestan no sólo símbolos sino emociones y pensamientos simbolizados.

Lo que está en juego aquí es si, desde una perspectiva etnográficaantropológica, las formas y contenidos performativos que cada carrera moral del vagabundo representa tienen el espíritu necesario para crear tradición. Pues, al parecer la existencia del vagabundo (y de cualquier persona) depende no sólo de las formas y contenidos que él mismo ( $y$ cada cual) ha sido capaz de crear por cuenta propia dentro de los límites de una comunidad de significados más o menos compartidos sino también de las formas y contenidos que, como miembro de esa comunidad de significados, ha podido incorporar a su historia y a su manera de ser.

La fuerza de esto último indica precisamente el valor de la tradición en la comprensión de la experiencia sociocultural. La importancia que tiene para los antropólogos la existencia de las tradiciones radica en que éstas permiten establecer precisamente 
cuando una proposición o un acto es o no cultural (Geertz, 1991; Spiro, 1992; Levy, 1992). Sin embargo, el carácter imprescindible de lo primero sugiere la posibilidad de un mundo sociocultural con su propia lógica en que la dimensión de la tradición no es muy visibic, a no scr que la tradición sea, para este caso, justamente aquello por lo cual el vagabundo puede vivir su vida, o sea, el estar más afuera que adentro de los convencionalismos y los estilos más colectivamente promovidos.

Sugiero que, en este punto, la noción antropológica de un mundo de experiencia posible puede verse amplificada a través del análisis de la vagabundancia y la existencia de los vagabundos. Al tensar algunas dimensiones de la experiencia sociocultural -el estar adentro/estar afuera, cl todo y la nada-, el vagabundo y la etnografía de su cuerpo, su rostro, sus acciones, sus palabras, sus muecas y al interior mismo de esas dimensiones -el contexto vital-, proporcionan algunas bases para la comprensión antropológica de problemas más profundos vinculados al relativismo cultural, la ineluctabilidad del horizonte lingüístico y el etnocentrismo. De una manera implícita e indirccta, estos problemas se han asomado a la indagación y parecen comenzar a abrirse...

\section{Mensaje final}

Para finalizar quisiera señalar que la sociedad sólo parcialmente ha vislumbrado aquello para 10 cual tiene menos preparación. Tanto la práctica como la visión de «correr contra la vida y contra Dios» requicre de la propia sociedad una manera distinta y más creativa para enfrentarias. El núcleo de las carreras morales de los vagabundos en Temuco ( $y$, en general, en las ciudades relativamente grandes del país) puede tener que ver con un modelo de sociedad que, al no lograr producir la integración y la participación, abandona, paradójicamente, a las personas a su propio destino: destino que culminará finaimente, como he tratado de mostrar, en fragmentación, desarraigo y desvinculación, cuando no en alienación y decadencia moral.

Alguien podrá preguntar ¿qué tiene que ver la sociedad en todo esto?, ¿por qué no cambia el vagabundo antes que la sociedad? El mensaje que quiero dar tiene que ver con que gran parte de los estigmas y las carreras estigmatizantes que, respectivamente abrazan y corren los individuos en sociedades como la nuestra, son construcciones simbólicas y, por lo tanto, son del dominio exclusivo de la historia de los hombres.
Por ello, cabe preguntarsc en relación a los vagabundos, como lo hizo alguna vez cierto psiquiatra en relación a otros estigmatizados -los locos-, si el problema de la integración y la inclusión no depende más bien del poder que establecen los propios estigmas, es decir, de construcciones con que el ser humano orienta su vida, estableciendo la definición de las personas y de las distintas situaciones. Si esto se reconoce como tal es porque quizás los propios símbolos de la exclusión son en gran medida una de las razones que impiden la participación y en general la comunicación sociocultural.

¿No será que los propios estigmas se basan en nuestras incapacidades para establecer comunicaciones coherentes con quienes han abrazado los atributos que los definen como estigmatizados?

\section{Bibliografía}

BERHO, M.. (1998), "Esbozo para una etnografia del vagabundo", en Revista CUHSO, Vol. 4, No 1, pp. 38-43, Centro de Estudios Socioculturales, Universidad Católica de Temuco, Chíle.

BEFHO, M. (1998), Condición sociocultural del vagabundo (adulto) de la ciudad de Temuco, con fines de reinserción social, Texto inédito, CESCORFOSAM, Temuco, Chile.

BOSING, W. (1994), El Bosco, Taschen.

FOUCAULT, M. (1994), Historia de la locura en la época clásica, Tomo 1, Fondo de Cultura Económica, México.

FOUCAULT, M. (1993), L.a vida de los hombres infames, Tusquets, Un:guay.

GEERTZ, C. (1991), La interpretación de las cuituras, Gedisa, México. GOFFMAN, E. (1979), Estigma. La identidad deteriorada, Amorrortu, Buenos Aires, Argentina.

GOFFMAN, E. (1993), La presentación de la persona en la vida cotidiana, Amorrortu, Buenos Aires, Argentina.

LE VINE, R. (1992), "Properties of culture: an ethnographic view", en Culture Theory. Essays on Mind, Self and Emotion, Shweder and Le Vine (ed.), Cambridge University Press, pp. 67-87.

LEVY, R. (1992), "Emotion, knowing, and culture", en Culture Theory. Essays on Mind, Self and Emotion,Shweder and Le Vine (ed.), Cambridge University Press, pp. 214-237.

ROSALDO, M. (1992), "Toward an anthropology of self and teeling", en Culture Theory. Essays on Mind, Self and Emotion, Shweder and Le Vine (ed.), Cambridge University Press, pp. 137-157.

SPIRO, M. (1992), "Some reflections on cultural determinism and relativism with special reference to emotion and reason", en Culture Theory, Essays on Mind, Self and Emotion,Shweder and Le Vine (ed.), Cambridge University Press, pp. 323-346.

TAUSSIG, M.(1995), "La reificación y la conciencia del paciente", en Un. gigante en convulsiones. El mundo humano como sistema nervioso en emergencia permanente, Gedisa, México, pp. 110-143. 